Brit. J. industr. Med., 1953, 10, 235.

\title{
THE IMPORTANCE OF THE TIME FACTOR IN THE MEASUREMENT OF DUST EXPOSURE
}

\author{
BY \\ B. M. WRIGHT* \\ From the Pneumoconiosis Research Unit of the Medical Research Council, Llandough Hospital, near Cardiff
}

(RECEIVED FOR PUBLICATION AUGUST 11, 1953)

Measurements of dust concentrations for the estimation of the danger of pneumoconiosis have been carried out in various industries for many years. In some, such as the South African gold fields, they have been made regularly for nearly $\mathbf{4 0}$ years, while in others, such as the British coal-mines, regular dust sampling on a national scale has only been carried on for about five years. In other industries again, such as foundries, normally only occasional samples are taken in an attempt to make some estimate of the likely danger of pneumoconiosis. In all this work the main emphasis has been placed on the factor of concentration and little is heard of the possible importance of the duration of exposure.

It is the purpose of this paper to attempt to arrive at an estimate of the relative importance of these two factors, since such an estimate is essential for the proper planning of dust sampling and dust suppression procedures. It must be emphasized that the discussion is concerned only with dusts which are of such low solubility that, if they are dissolved at all, their rate of solution is very low compared with their rate of inhalation and deposition in the lung. Examples of such dusts are quartz and coal, which between them produce most of the serious cases of pneumoconiosis in this country (Ministry of National Insurance, 1952).

For the purpose of this discussion we shall assume, as a first approximation, that the severity of a pneumoconiosis due to a particular dust will be dependent upon the amount of that dust in the lungs. This is obviously not entirely true, but it is necessary to make some assumptions in order to be able to define the problem. Such evidence as exists (Badham

* The author was a delegate to the International Labour Office * The author was a delegate to the International Labour Office
Conference on the Prevention and Suppression of Dust in Mining, Tunnelling, and Quarrying at Geneva in December, 1952. Many of the statements in this paper are based on discussions with mining engineers and others at this Conference. and Taylor, 1938, 1940 ; King and Nagelschmidt, 1945) suggests that even when there are marked changes in pathology, e.g. from simple pneumoconiosis to massive fibrosis in coal-workers' lungs, there is a rough correlation between the amount of dust in the lungs and the severity of the disease. Our problem therefore is, What are the relative effects of concentration and time on the rate of inhalation and the retention of dust in the lungs?

In the absence of any other information, the natural assumption would seem to be that concentration and time are equally important, i.e., that the amount of dust retained in the lungs is proportional to the product of the two, so that an exposure to a concentration $\mathrm{C}$ for a time $\mathrm{T}$ will have the same effect as an exposure to a concentration $2 \mathrm{C}$ for a time $\frac{T}{2}$. This, which might be termed the "average" hypothesis, has been adopted by all workers who have tried to correlate dust exposure with disease incidence, and it is interesting to note that, in spite of the inevitable imperfections in their measurements, all workers who have made such studies have found a reasonable correlation (Bedford and Warner, 1943 ; Dreessen, DallaValle, Edwards, Miller, Sayers, Easom, and Trice, 1938 ; Dreessen, DallaValle, Edwards, Sayers, Easom, and Trice, 1940 ; Flinn, Dreessen, Edwards, Riley, Bloomfield, Sayers, Cadden, and Rothman, 1939; Flinn, Seifert, Brinton, Jones, and Franks, 1941 ; Roach, 1953 ; Sayers, Bloomfield, Dalla Valle, Jones, Dreessen, Brundage, and Britten, 1935).

Even if we knew nothing at all about the mechanism of dust inhalation and retention, the fact that the "average" hypothesis is simple would be in itself a strong point in its favour; although it must be recognized that a simple hypothesis seldom turns out to be entirely satisfactory, it is an essential principle in research that the simplest 
hypothesis that will fit all the known facts should be adopted. It might be thought, therefore, that in the absence of any contrary evidence the "average" hypothesis would be adopted as a working assumption by those concerned with dust sampling for the prevention of pneumoconiosis. But in my experience there is a widespread belief that this hypothesis is incorrect; that high concentrations are disproportionately important and lead to a much greater accumulation of dust in the lungs than would be expected on the simple " average" hypothesis. This belief is clearly stated by Drinker and Hatch (1936), who say :

"A concentration of $\mathbf{4 0}$ million particles per cu. $\mathrm{ft}$. in a granite shed, for example, is probably more than twice as dangerous as one of 20 million."

They emphasize repeatedly the necessity for frequent sampling in order to detect " dust floods", and maintain that to suppose that prolonged exposure to low concentrations is just as serious as short exposure to heavy concentrations is contrary to a fundamental " law of physiology", namely that :

" a sub-threshold stimulus (dust inhalation) for a long time produces no reaction, whereas a relatively brief superthreshold stimulus may cause a reaction."

This "law" is really only a description of the way in which certain physiological mechanisms behave. It will be seen, when we come to consider the mechanisms of dust inhalation and retention, that they are not of this type so that the "law" is inapplicable.

In view of their emphatic rejection of the simple average hypothesis, it is unfortunate that the only actual evidence against it quoted by Drinker and Hatch is a statement by Mavrogordato (1923) that :

"Lesions of silicosis in a mild degree can be produced in an animal by $30 \mathrm{hr}$. exposure to intense dust clouds, and one is inclined to suspect that it is intermittent exposure to relatively dense clouds that is the deciding factor in producing the disease in susceptible human subjects."

Apart from the extremely tentative nature of his conclusions, as indicated by the words which have been italicized, there is no record in Mavrogordato's works $(1918,1922,1923,1926,1940)$ that he ever actually compared the results of high and low concentrations in animals in such a way as to demonstrate that the higher concentrations were disproportionately dangerous.

Another eminent authority on pneumoconiosis, L. U. Gardner, has stated (Gardner, 1941) :

"Atmospheric concentration of dust must be high enough to overcome the efficiency of the upper respiratory protection and permit an excess of particles to enter and accumulate in the lungs."
In view of the deservedly high reputation of these authors, it is not surprising that the simple average hypothesis is rejected by many mining engineers, who insist on the necessity of frequent sampling to detect "peaks" of dust production. For instance, Flugge-de-Smidt (1951) remarks :

"Is it any advantage to obtain average dust counts? I would say decidedly not.

"The filtration processes of the human body are capable of filtering dust out of the air when that dust is only present up to a certain concentration, say for sake of argument, 300 particles per c.c. Should a person encounter a dust cloud of, say, 1,000 particles per c.c. as registered by a konimeter, then the human body can only cope with, say, 300 particles and the remaining 700 particles enter the lungs. The rate of breathing is another factor affecting the filtering process of the human body. Believing this to be fundamentally correct, I have always stressed the danger of occasional peaks in dust curves as being of prime importance. An average of, say, 200 particles per c.c. over half an hour can have been more dangerous than an average of 300 particles per c.c. if in the first case a number of peaks occurred, and in the second case a smoother curve was obtained. Having taken thousands of dust samples in development ends, I was impressed first of all with the enormous variations in dust production during normal drilling and the very high peaks obtainable when for instance, a machine was collaring. We need an instrument to 'snapsample' such peaks and make us aware of such dangers. The thermal precipitator or any other similar instrument is utterly incapable of giving us that information."

Similarly, the National Coal Board in their memorandum "The Sampling of Air-borne Dust for the Testing of 'Approved Conditions " " (1949), which is the basis of the present sampling practice in British coal mines, state :

"The Board have accepted that the standards are to be interpreted not as defining the allowable average concentration of dust over the whole period of a working shift, but the average dustiness during periods of maximum dust production." [Their italics.]

They go on to point out that these periods will vary from colliery to colliery so that it appears that they consider the level of the dust concentrations more important than their duration, as they do not propose to take variations in the latter into account.

Recently, McLaughlin (1953) has reaffirmed that " intermittent exposure to higher concentrations of dust may be more dangerous than exposure to lower concentrations over a longer period", although he cites no evidence in support of this statement.

Unfortunately, this belief in the disproportionate importance of high concentrations, which may for convenience be termed the "peak" hypothesis, has never been sufficiently clearly defined for its 
validity to be tested; but an essential feature of it appears to be a concept of a threshold mechanism which breaks down under the strain of high concentrations and either allows an excessive amount of dust to enter the lungs or causes an unusual amount to be retained. In view of the popularity of the " peak" hypothesis and its important effect on dust sampling and dust suppression practice, it seems worth while to examine this concept in the light of what is known about the mechanism of dust inhalation and retention to see whether there is any evidence for the existence of such a threshold.

The Mechanism of Dust Inhalation and Retention

Our knowledge of the mechanisms of dust inhalation and retention has recently been reviewed by Davies (1949) and it will not be considered in more detail here than is necessary to decide the merits of the two theories.

Briefly, it is believed that dust enters the respiratory tract suspended in the air breathed, but particles over a certain size (about $5 \mu$ diameter) are deposited on those parts of the respiratory tract which are covered with ciliated epithelium and are removed from the lungs completely in, at most, a few hours. Smaller particles reach the alveoli and a proportion of them, depending on their size and the breathing pattern, are deposited on the alveolar walls. The remainder are exhaled again in the expired air. A small proportion of these exhaled particles may be deposited on the ciliated epithelium, but in any case they will not be retained in the lung. Particles deposited in the alveoli can probably only be removed from there by phagocytosis. (A number of workers have suggested (Gross and Brown, 1952; Mottura, 1952) that particles are carried out of the alveoli by simple lymph flow, but the exact mechanism by which dust particles are transported does not affect the present argument.)

It appears that only a proportion of the dustcontaining phagocytes migrate out on to the ciliated epithelium; the remainder are carried into the lymphatic system and are deposited in the interstitial tissue of the lung. These interstitial deposits are more or less permanent, and constitute the condition of pneumoconiosis. It is evident that, as so defined, pneumoconiosis is a condition by which, to a greater or less extent, all adults are affected, and it is only when the dust is toxic, or if not toxic, excessive in quantity, that a disease results. It is obvious that there is room for great difference of opinion about the stage at which the condition becomes a disease.

In order to be able to examine the "average" hypothesis in the light of this account of the mechanism of dust inhalation and retention, it is necessary to state it as clearly as possible. This hypothesis may therefore be re-stated as follows : The rate of accumulation of dust in the lung is directly proportional to the concentration of respirable dust in the air. Thus the quantity of dust retained in the lung after exposure will on the average be directly proportional to the product of the concentration and the time of exposure.

Is there anything in our picture of the mechanism of dust inhalation and retention to cause us to reject such a belief, and make the rate of accumulation of dust no longer directly proportional to the concentration of respirable dust in the air?

Before we can answer this question, we must first consider what we mean by "the concentration of respirable dust". For practical purposes, this can be taken to mean the concentration of particles and aggregates below $5 \mu$ in diameter, since only they have an appreciable chance of being retained in the lung. It is sometimes suggested that the larger particles could play a part by swamping the ciliated epithelium and preventing their own removal, but this seems unlikely, since the epithelium is very efficient, and even if temporarily swamped during a severe exposure could recover and clean itself as soon as the exposure was over (Barclay, Franklin, and Macbeth, 1938; Barclay, Franklin, and Prichard, 1942). In any case, the most important effect of a high concentration of coarse particles would be to produce coughing, which would probably increase the rate of removal of dust from the upper respiratory tract.

Although it is possible that continued exposure to coarse dust might cause permanent damage to the ciliated epithelium, such an effect has not been recorded, and Cummins (1936) noted that in coal miners of many years' standing with severe pneumoconiosis the ciliated epithelium of the bronchi remained intact. Moreover, it must be realized that the results of such damage, though serious, would not constitute pneumoconiosis as it has been defined. The most striking effect would be chronic bronchitis, with chronic cough, which in fact is not an early symptom of pneumoconiosis and may be absent even in the presence of severe dyspnoea and marked $x$-ray changes (Gooding, 1946).

We are left then with the consideration of the effect of concentration of the respirable dust on its inhalation and retention in the alveoli. There appears to be no conceivable mechanism by which concentration could disproportionately affect the rate of dust inhalation. It is true that with manual working there is commonly a correlation between high dust concentrations and high breathing rates, 
but this correlation is not causal and breaks down seriously when machine methods of mining and dust suppression are introduced. On the other hand, it is conceivable that, as is often suggested, high concentrations could increase the rate of retention by overloading the phagocytes and so reducing the proportion which escaped to the ciliated epithelium. This seems a plausible suggestion, but in the first place all those who have carried out dust inhalation experiments on animals, using concentrations much higher than occur naturally, are familiar with the fact that the rate of phagocytosis is always equal to the rate of deposition in the alveoli (Barclay and others, 1942 ; Carleton, 1934). Even after exposure to these very high concentrations, it is rare to find more than a few extracellular dust particles in the alveoli even within a few minutes of exposure, because the actual rate at which dust is deposited in the alveoli is low (about two particles a minute per alveolus in a dust concentration of 10,000 particles $/ \mathrm{ml}$.) and well within the capacity of the phagocytes. Such few observations as have been made in man confirm this finding (Heppleston, 1953 ; Policard, 1952). It can therefore be said that dust concentrations high enough to overload phagocytes seriously will seldom occur in practice. It has yet to be shown, moreover, that slowing their rate of migration would alter the proportion of phagocytes migrating to the ciliated epithelium as compared with those entering the interstitial tissue. It is true that very high concentrations of highly toxic siliceous dusts may lead to paralysis of the phagocytes so that they accumulate in the alveoli, but this is an extreme case and leads to acute alveolar silicosis, a rare condition.

From these considerations, it appears that there is no definite physical or physiological basis for the concept of a threshold below which the lungs free themselves completely of dust, as has been claimed (Cummins, 1927); on the contrary, the lungs would be expected to start collecting dust from the first breath, and continue to do so throughout their owner's life, and it is of course well known that this is the case ; the lungs of a child are pink, while those of an adult city dweller are grey. On the other hand, there is what might be termed an "observer" threshold, resulting from the fact that a certain minimum amount of dust in the lungs is required to produce perceptible radiological or physiological change. Since a man's working life is limited, dust concentrations below a certain level will not give rise to diagnosable pneumoconiosis at all.

Although, therefore, there are a number of possible mechanisms which might lead to the lungs being unduly affected by high concentrations, they do not seem very probable, and there certainly seems no justification for assuming their existence in the absence of evidence.

The popularity of the "peak" hypothesis, however, probably does not depend only on the weight of the authorities quoted in its favour; there are three reasons why it might appeal, especially to practical mining engineers. In the first place, men who are exposed to high concentrations of coarse dust, even for short periods, may cough and subsequently bring up dust-stained sputum. They confuse these manifestations with the symptoms of pneumoconiosis, although, as we have seen, they are in fact quite different, and therefore tend to attach great importance to such exposures, while ignoring their exposure to lower concentrations or finer dust which produce no immediate obvious effect.

Secondly, the "peak" hypothesis fits in very well with a sampling procedure which grew up for quite different reasons. An engineer who is trying to suppress dust feels it natural to look for the highest concentrations and deal with them first.

Thirdly, and an important reason for its popularity in the coal industry, there is a confusion with the fire damp danger. It is often said that dust should be treated like gas ; if this is meant to imply either that it is as important a danger as gas, or that its aerodynamic behaviour is similar, few could dispute such a simile. But the danger of gas only arises above a certain concentration level and is immediate; safety can only be achieved by ensuring that the danger level is never reached, even momentarily, and therefore a policy of continually looking for the highest concentrations is perfectly proper. In the case of dust, however, in view of the relatively long period of exposure which is necessary before any ill effects are produced, this policy is inappropriate unless it is believed that peak concentrations are especially significant, and not simply a proportional contribution to the average.

\section{Practical Consequences}

We are left then with the conclusion that there is nothing in our knowledge of the relationship between dust exposure and disease incidence, or the mechanism of inhalation and retention of dust, to substantiate the "peak" hypothesis or to conflict with the "average" hypothesis. It remains to be considered whether there should be any modification of the present sampling and dust suppression practices in the light of this conclusion, since the present practices are so largely influenced by the "peak" hypothesis. 
It is frequently maintained that there are several reasons for dust sampling (e.g. Long, 1953) and that a different technique is appropriate for each of them. The reasons given are : (1) Looking for sources of dust production and studying the effectiveness of dust suppression methods ; (2) routine control of dust conditions to comply with regulations; and (3) scientific research into the relation between dust exposure and disease incidence. It is claimed that the first two are the province of engineers and officials, who will decide for themselves which methods are most appropriate, while the third is the job of the medical research worker, who may use more refined methods if he chooses.

It is clear, however, that this attitude is illogical ; in so far as the engineers and officials are concerned with the same problem as the medical research worker, namely dust as a potential cause of pneumoconiosis, they must measure the dust exposure in fundamentally the same way; that is to say, they must take into account not only the dust concentrations but the duration of the men's exposure to them. It is true that when conditions are bad very rough and ready methods are satisfactory; for instance, the fact that an instrument such as the konimeter will only give the reading " too many to count" in excessively high concentrations does not much matter for control purposes, while it causes considerable difficulty when attempting to measure exposure accurately. But as soon as dust conditions are fairly satisfactory, any further improvement will depend on the use of more precise methods of measurement so that dust suppression measures can be most economically employed. It is at this point that the engineers begin to ask the research workers about "safe limits", and it is then that their interests become identical. The main purpose of dust disease correlation studies is to determine safe limits, and they must be determined with the instruments and sampling techniques that are going to be used as a routine. As we have seen, as a result of the widespread belief in the "peak" hypothesis, most routine sampling is carried out in such a way as to take little or no account of the duration of exposure. Its great disadvantage as a basis for action is that it requires a much more expensive and laborious sampling procedure than the "average" hypothesis and yet, owing to its lack of definition, the information so laboriously obtained is in fact not used except by pooling and averaging it. To measure the average by taking large numbers of short-term samples and then averaging them is wasteful of labour, if automatically averaging instruments are available, unless the individual samples can be used in some way. It will be said, of course, that they can be used to detect important sources of dust production, but it is hard to see how the importance of these can be assessed unless the duration as well as the concentration of the dust production is measured. Also, the individual samples can only be used for the detection of dustproducing processes if careful records are kept both of the dust concentrations and of the dust-producing processes. Thus an automatic, continuously recording dust sampler such as has been described by Lloyd and Winder (1951) for coal mines is not of much value unless its record is accompanied by a corresponding record of the mining procedures, when the man who is keeping the record might just as well be operating the sampler.

It would seem, therefore, to be a more practical procedure in most cases, if the average is accepted as the proper index, to measure it directly in the most convenient way, and leave the problem of deciding which are the important sources of dust production to the man on the spot. To try to help him by giving him a snap sampling instrument such as a "tyndallometer" (Broomhead and Burdekin, 1953), which yields an immediate reading of dust concentration, may be as much a hindrance as a help, as it will tend to direct his attention mainly to sources of high concentration and cause him to neglect those of lower concentration but of longer duration, and therefore equally or more important as contributions to the average. It is true that there appears to be a correlation between high peaks and high averages (Oldham, 1953), but if the average can be measured directly and easily it is unsatisfactory to measure peaks and rely on a correlation which may not be constant. The danger of using a short-term instrument in addition to an averaging sampler is that the operator will tend to attach more weight to the results of his short-term instrument than to the average, and where it differs to ignore the latter.

Apart from its effect on dust sampling practice, the belief in the "peak" hypothesis is liable to cause some misdirection of energy in the practice of dust suppression. For instance, great stress is laid on the danger arising from such processes as shot firing or the blowing out of drill holes. In the absence of good ventilation these processes, which undoubtedly produce a large quantity of dust and momentarily very high concentrations, are certainly an important source of danger. But once ventilation is established the dust produced is rapidly diluted and carried away, and the danger from these processes is thus correspondingly reduced, and may well make them less important as a source of dust 
production than some less dramatic and conspicuous process, such as for example shovelling, which is carried on for much longer periods.

\section{Conclusion}

In conclusion, then, we may sum up by saying that the present widespread belief in an ill-defined "peak" hypothesis is not supported by any evidence ; it leads to a laborious sampling technique and, if the " average " hypothesis is correct, may lead to some misdirection of energy in dust suppression. It cannot be stated with certainty that the "average" hypothesis is perfectly correct ; the most that can be said is that it is a simple and reasonable hypothesis which does not conflict with anything that is known about the mechanism of dust inhalation and retention; so far as it has been possible to check it, it has been found to correspond with the observed facts about the relation between dust exposure and disease incidence. Since it leads to a much simpler, cheaper and more satisfactory dust-sampling technique, there seems to be every reason for adopting it as a working hypothesis in place of the "peak" hypothesis, which at present holds the field to such a large extent.

\section{Summary}

Two hypotheses of the relative importance of time and concentration in the production of pneumoconiosis are widely accepted. On one, the " peak" hypothesis, high concentrations are believed to be disproportionately important, while on the other, or " average" hypothesis, concentration and time are believed to be equally important.

The evidence for and against the two hypotheses is considered and it is concluded that the " average" hypothesis is to be preferred.

The effects of this conclusion on dust sampling and dust suppression practice are discussed.
I am indebted to my colleagues at the Pneumoconiosis Research Unit for their valuable criticisms during the preparation of this paper.

\section{REFERENCES}

Badham, C., and Taylor, H. B. (1938). Report of the DirectorGeneral of Public Health, New South Wales, for 1936, p. 100 General of Public Health, Ne

Barclay, A. E., Franklin, K. J., and Macbeth, R. G. (1938). Amer. J. Roentgenol., 39, 673 .

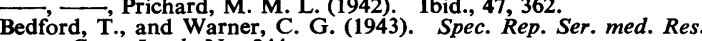
Coun. Lond., No. 244.

Broomhead, G., and Burdekin, J. T. (1953). Ministry of Fuel and Power (Safety in Mines Research Establishment) Res. Rep. Now 61 .

Carleton, H. M. (1934), Proc. roy. Soc. Lond., ser. B., 114, 513.

Curleton, H. M. (1934). Proc. roy. Soc. Lond.,

Cummins, S. L. (1927). J. Path. Bac.

Davies, C. N. (1949). British Journal of Industrial Medicine, 6, 245. Dreessen, W. C., DallaValle, J. M., Edwards, T. I., Miller, J. W. Sayers, R. R., Easom, H. F., and Trice, M. F. (1938). U.S. Publ. Hith Bull., Wash., No. 241.

,$- \quad$ Sayers, R. R., Easom, H. F., and Trice, M. F. 1940). Ibid., No. 250.

Industrial Dust, McGraw-Hill, New York.

Flinn, R. H., Dreessen, W. C., Edwards, T. I., Riley, E. C., Bloomfield, J. J., Sayers, R. R., Cadden, J. F., and Rothman, S. C. (1939) J. J., Sayers, R. R., Cadden, J. F., and

Seifert, H. E., Brinton, H. P., Jones, J. L., and Franks, R. W. (1941). Ibid., No. 270.

Flugge-de-Smidt, R.A.H. (1951). in (discussion on) Beadle, D. G., J. chem. Soc. S. Afr., 51, 283.

Gardner, L. U. (1941). The Pneumoconioses, in Nelson New LooseLeaf Medicine, 3, 481. Thomas Nelson \& Sons, New York.

Gooding, C. G. (1946). Lancet, 2, 891.

Gross, P., and Brown, J. H. U. (1952). Amer. J. clin. Path., 22, 821

Heppleston, A. G. (1953). Personal communication.

King, E. J., And Nagelschmidt, G. (1945). Spec. Rep. Ser., med. Res. Coun. Lond., No. 250

Lloyd, H., Winder, G. E., (1951). Ministry of Fuel and Power (Safety in Mines Research Establishment) Res. Rep. No. 29

Long, W. M. (1953). British Journal of Industrial Medicine, 10, 241.

Mavrogordato, A. (1918). J. Hyg. Camb., 17, 439.

-(1922). Publ. S. Afr. Inst. med. Res., No. 15.

(1923) Ibid., No. 17.

(1926). Ibid., No. 19.

- (1940). J. chem. Soc. S. Afr., 40, 326.

Ministry of National Insurance (1952). Third Annual Report, for the year 1951. H.M.S.O., Cmd. 8635 .

Mottura the (1952) British Journal of Industrial Medicine, 9, 65. Mottura, G. (1952). British Journal of Indust

National Coal Board (1949). Circular : The Sampling of Air-borne Dust for the Testing of "Approved Conditions"

Oldham, P. D. (1953). British Journal of Industrial Medicine, 10, 227

Policard, A. (1952). Ibid., 9, 108.

Roach, S. A. (1953). British Journal of Industrial Medicine, 10, 220.

Sayers, R. R., Bloomfield, J. J., DallaValle, J. M., Jones, R. R., Dreessen, W. C., Brundage, D. K., Britten, R. H. (1935) U.S. Publ. Hith Bull., Wash., No. 221 . 